\title{
Degradation of Glycine and Alanine on Irradiated Quartz
}

\author{
Maciej Pawlikowski • Aleksandra Benko • \\ Tomasz P. Wróbel
}

Received: 20 January 2013 / Accepted: 1 March 2013 /

Published online: 28 March 2013

C The Author(s) 2013. This article is published with open access at Springerlink.com

\begin{abstract}
Recent researches suggest participation of minerals in the formation of life under primordial conditions. Among all of the minerals, quartz seems to be one of the most probable to take part in such processes. However, an external source of energy is needed, e.g. electric discharge. A device simulating the proposed conditions was designed and was used to simulate prebiotic conditions. Investigation of processes occurring during the stimulation of quartz with electric discharge was studied by means of Ultraviolet-visible (UV-VIS) spectroscopy, in order to monitor the generation kinetics of free radicals. Additionally, infrared spectroscopy was applied to identify chemical reaction products created in a solution of alanine or glycine, in the presence of quartz treated with electric discharge. Formation of increased amounts of free radicals, compared to experiments performed without quartz and/or amino acid, is reported, along with identification of possible degradation products of alanine. No synthetic reactions were observed.
\end{abstract}

Keywords Quartz · Free radicals · Amino acids · Electric discharge · FTIR-ATR · UV-VIS

\section{Introduction}

In recent years many scientists have independently proven that minerals promote polymerization of amino acids into protein-like structures, support development of lipidic layers and

Electronic supplementary material The online version of this article (doi:10.1007/s11084-013-9328-3) contains supplementary material, which is available to authorized users.

M. Pawlikowski

Laboratory of Biomineralogy, Department of Mineralogy, Petrography and Geochemistry, Faculty of Geology, Geophysics and Environment Protection, AGH-University of Science and Technology, A.

Mickiewicz 30 Ave, 30-059 Krakow, Poland

A. Benko $(\square)$

Department of Biomaterials Science, Faculty of Materials Science and Ceramics, AGH-University of Science and Technology, A. Mickiewicz 30 Ave, 30-059 Cracow, Poland

e-mail: akbenko@gmail.com

T. P. Wróbel

Faculty of Chemistry, Jagiellonian University, Ingarden 3 St, 30-060 Cracow, Poland 
stimulate and serve as scaffolds for the self assembly of RNA nucleotide(Lambert 2008; Hazen 2006). Among all of the minerals known to mankind, quartz seems to be one of the most probable to participate in prebiotic chemistry. Apart from being the most common mineral on Earth, many distinctive features, such as homochirality, piezoelectricity and the ability to form free radicals under mechanical activation seem to support its plausible role in the formation of life (Damm and Peukert 2009).

As a stable mineral, quartz does not possess a high potential to initialise, alter or steer any chemical process. In order to do so, some source of external energy is needed (Pross 2004). A highly probable source of such energy seems to be electric discharge. In the small water pond, filled with quartz crystals and amino acids, such an occurrence could cause reverse piezoelectric effects in the crystal, hydrolysis of water and ozone generation (Sahni and Locke 2006; Ueda et al. 2009). These factors could influence molecular structure and/or constitution of organic compounds.

Fourier transform infrared spectroscopy (FTIR) has proven in the past to be a very powerful analytical technique, especially when used in Attenuated Total Reflection (ATR) mode (Kazarian and Chan 2006). It can be successfully applied as a method of analysis for solid samples (Wróbel et al. 2011). Low sample amount requirement, no additional sample preparation and ability to measure aqueous solutions are the greatest advantages of the method. However, the true potential of the technique lays in the application to more demanding samples, such as single cells (Wróbel et al. 2012).

The aim of the work presented here was to examine the hypothesis that quartz, under the influence of electric discharge, could modify the molecular constitution and/or structure of simple amino acids. The idea that dipeptides and polypeptides can be created under such condition was investigated. Among 22 proteinogenic amino acids, two of the simplest structures were chosen - alanine and glycine. Short side chains and no inorganic substituents should simplify the eventual reaction, increasing the chance for better understanding the whole process. Chemical changes in the tested compounds were monitored in time using the FTIR-ATR method, as well as by UV-VIS spectroscopy and the use of DPPH as a scavenger.

\section{Materials}

Quartz

Quartz of highest purity was obtained from the Jagielowa mine (near Strzelin, Poland), crushed and sieved, in order to obtain a fraction, named large quartz (LQ), with grains of diameter size between 0.1 and $2 \mathrm{~mm}$.

Quartz purity was evaluated using FTIR-ATR spectroscopy (an infrared spectrum is shown in Online Resource 1, S.M. 1) Only bands attributable to quartz can be seen, which, along with band intensity proportions, prove the crystallographic structure and relatively low contribution of defects (Apopei et al. 2011; Saikian et al. 2008; Shneider 1978; Bobrowski and Holtzer 2010; Shoval 1991; Hlavay et al. 1978.)

\section{Amino Acids}

Both alanine and glycine of $96 \%$ purity were purchased from Sigma Aldrich. For evaluation of structural changes, both amino acids were dissolved and diluted in distilled water to $0.019 \mathrm{M}$ and $0.011 \mathrm{M}$ concentrations, respectively. For free radicals' detection, glycine solution of $0.015 \mathrm{M}$ concentration was prepared. 


\section{$\mathrm{DPPH}$}

Free radical generation and kinetics under influence of electric discharge and piezoelectric quartz were assessed using 2, 2-diphenyl-1-picrylhydrazyl (DPPH Sigma Aldrich, molar mass $394.32 \mathrm{~g} / \mathrm{mol}$ ) as a scavenger. DPPH was dissolved in methanol (J.T. Baker, $1112231002,99.99 \%$ purity) and diluted with water to concentration of $3 * 10^{-5} \mathrm{M}$ (abbreviated $\mathrm{DPPH}_{\mathrm{s}}$ throughout the article).

\section{Methods}

\section{Electric Discharge Apparatus}

An especially designed, custom-made, electric discharge device was used (Fig. 1). The apparatus was previously used by one of the authors (Pawlikowski 2012). The instrument is equipped with an electrode connected to a high voltage (approx. $50 \mathrm{kV}$ ) generator. The sample is put in the rotating reaction container (with a base made out of copper plate, used to create the proper electrical potential difference) and exposed to an electric discharge at the rate of two strikes per second.

\section{Detection and Kinetics of Free Radical Generation}

The experiments were based on methods proposed by Damm and Peukert (2009), using DPHH as a free radical scavenger. DPPH is a highly stable free radical, which, after dissolving in alcohol, forms a purple mixture, exhibiting two bands of maximum absorption at 511 and $325 \mathrm{~nm}$. The reaction with free radicals results in bleaching and can be easily monitored using a UV-VIS spectrometer. Rate of DPPH bleaching depends on the rate and the amount of generated free radicals.

All UV-VIS measurements were performed using a Perkin Elmer spectrometer, model Lambda 35. Spectral range was set to 200-1,000 nm. Disposable, 1.5-3 ml PMMA (Poly methyl methacrylate) cuvettes were used.

In order to evaluate free radical generation under the experimental conditions, four separate tests, using four different combinations of compounds were conducted:

a) $1.5 \mathrm{ml}$ of $\mathrm{DPPH}_{\mathrm{s}}$ and $6 \mathrm{ml}$ of water;

b) $1.25 \mathrm{ml}$ of $\mathrm{DPPH}_{\mathrm{s}}$ and $5 \mathrm{ml}$ of glycine solution

c) $1.5 \mathrm{ml}$ of $\mathrm{DPPH}_{\mathrm{s}}$ and $6 \mathrm{ml}$ of water with LQ

d) $1.25 \mathrm{ml}$ of $\mathrm{DPPH}_{\mathrm{s}}$ and $5 \mathrm{ml}$ of glycine solution with LQ

Each mixture was put in the reaction container of the electric discharge generator and exposed to electric discharge for $55 \mathrm{~min}$. Every $5 \mathrm{~min}$, the electric discharge apparatus was stopped, $2 \mathrm{ml}$ of the solution was pipetted, put in the disposable cuvette and its UV-VIS spectra was collected. After the data acquisition, the content of the cuvette was put back in the reaction container and the electric discharge apparatus was turned back on.

\section{Reaction Products Assessment}

Infrared spectral data was collected using a commercial Bruker FTIR-ATR spectrometer (Alpha equipped with Platinum ATR QuickSnap ${ }^{\mathrm{TM}}$ sampling module with a diamond ATR crystal for 


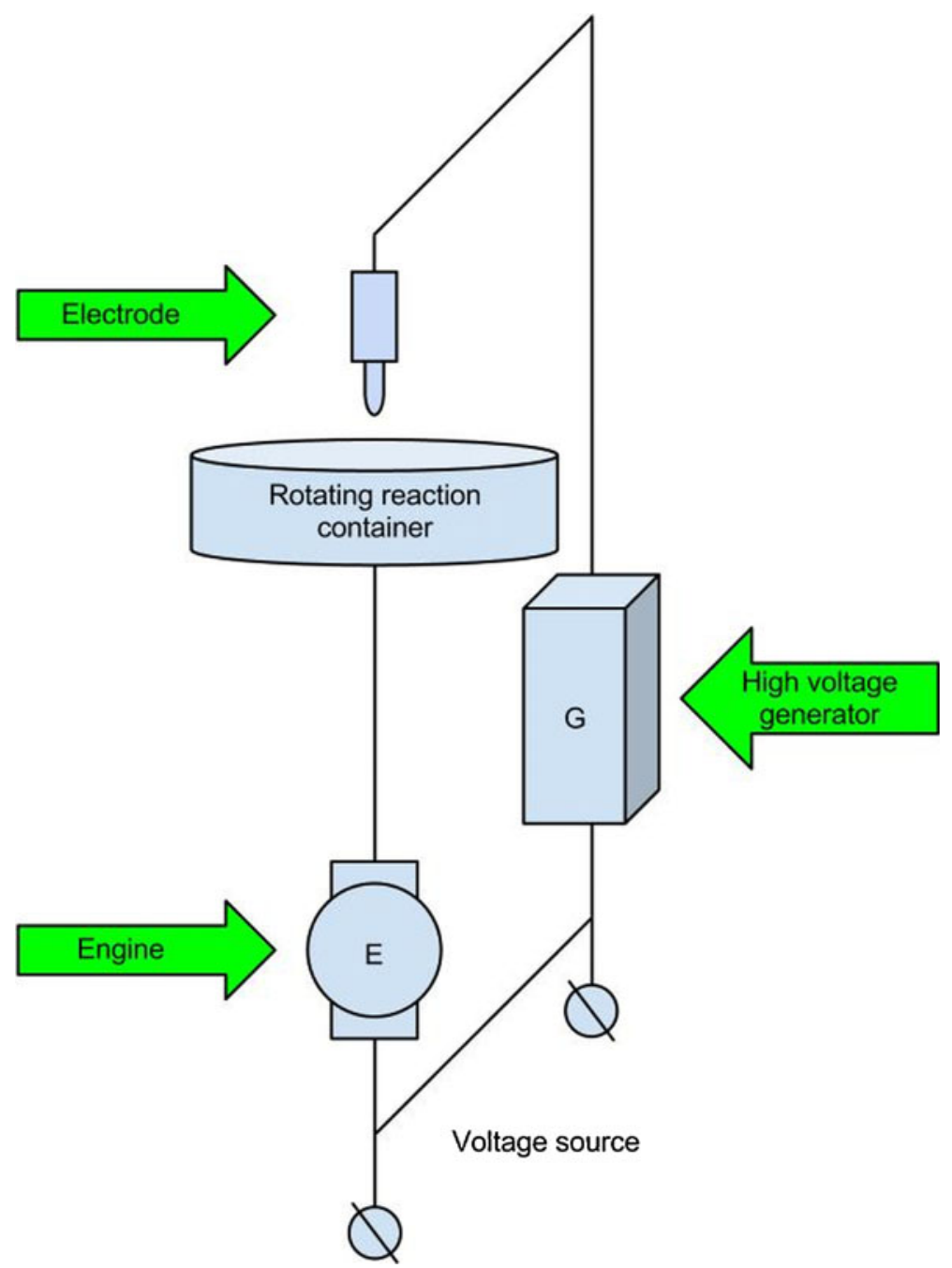

Fig. 1 Scheme of the electric discharge apparatus

solids and liquids, A220/D-01). Spectral range was set to 4,000-400 $\mathrm{cm}^{-1}$, number of scans128, as a background a clean ATR crystal was used. Experiments were performed for both amino acids separately with LQ in the reaction container and the blank test was performed using glycine without quartz. Reaction mixture was exposed to electric discharge for $70 \mathrm{~min}$ and every $10 \mathrm{~min}$ approx. $0.5 \mathrm{ml}$ of the solution was pipetted and measured using FTIR-ATR spectrometer. After $70 \mathrm{~min}$, the samples were filtered, in order to eliminate the quartz from the solution, and dried at room temperature and pressure. Resulting crystals were also analysed on the FTIR-ATR device.

\section{Data Treatment}

All infrared spectra were analysed and handled using OPUS 6.0 and EssentialFTIR software. No ATR corrections for dispersion and depth penetration were performed- 
the outcome data were not compared to any standard FTIR spectra. Presented spectral plots were created using Origin 8.6. UV-VIS spectra were analysed using Specwin32.

\section{Results and Discussion}

\section{Free Radicals}

Free radical formation in all of the reaction mixtures was proven by DPPH bleaching. With time, the value of both maxima of absorption bands in UV-VIS spectra decreases gradually (Online Resource 1, S.M. 2), therefore it can be assumed that the reaction of DPPH recombination is strictly time-dependent.

In order to compare the rates of DPPH bleaching in each mixture, reaction rate constants were calculated, assuming first-order reaction kinetics. Values of both absorption maxima are strictly correlated, the results for band at $540 \mathrm{~nm}$ are presented here. All spectra were fitted manually (as in Online Resource 1, S.M. 3). Absorption values were determined using program functionality.

Reaction rate constant (k) was calculated using Eq. 1.

$$
\operatorname{In} \frac{\mathrm{I}}{\mathrm{I}_{0}}=-2 \mathrm{kt}
$$

Equation 1 Rate constant calculation. I - absorbance instantaneous value, $\mathbf{I}_{0}-$ absorbance value at $\mathrm{t}=0, \mathrm{t}-$ time $[\mathrm{s}]$

$\ln \frac{\mathrm{I}}{\mathrm{I}_{0}} \quad$ values plotted against time are presented in Fig. 2. Highest rate of reaction is represented by mixture of quartz and glycine $\left(6.6 \cdot 10^{-3}\left[\mathrm{~s}^{-1}\right]\right)$ nearly two times lower rate is obtained for the blend of water with quartz $\left(3.6 \cdot 10^{-3}\left[\mathrm{~s}^{-1}\right]\right)$. Reaction rates for both mixtures without quartz (pure water solution and glycine in water solution) are significantly lower $\left(\left(3.0 \cdot 10^{-3}\left[\mathrm{~s}^{-1}\right]\right)\right.$ and $\left(2.2 * 10^{-3}\left[\mathrm{~s}^{-1}\right]\right)$, respectively).

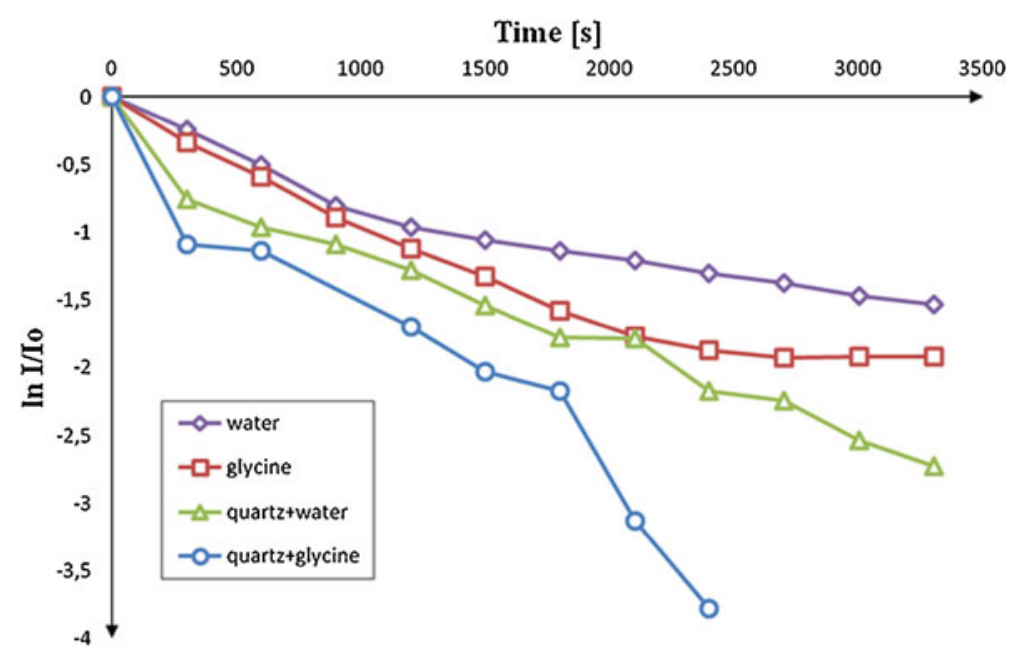

Fig. 2 Kinetics of the free radicals generation 
Therefore, the presence of glycine in water does not influence the rate of radical generation as much as the presence of quartz. Additionally, it seems that a combination of both factors enhances the reaction rate significantly - almost twofold. Considerably lower and similar reaction rates of the both tests performed without quartz can be ascribed to free radicals originating only from water hydrolysis (Sahni and Locke 2006). Possibly, additional factors provoke different pathways of radical generation, including initiation and propagation. The mechanism of such reaction can possibly be similar to the one suggested by Damm and Peukert (2009).

\section{Reaction Products Assessment}

The time dependent measurements of alanine solution subjected to electric discharge with quartz can be seen in Online Resource 1, S.M. 4, however, the differences observed in the spectrum are possibly attributed mostly to quartz (Apopei et al. 2011; Saikian et al. 2008; Shneider 1978; Bobrowski and Holtzer 2010). It was concluded that under the electric discharge, causing piezoelectric tensions, quartz disintegrates into very small pieces that obscure the analysis of the solutions. Therefore, measurements of the crystallites of the whole reaction mixture were assumed to be more accurate for the reaction interpretation.

A blank test of glycine solution without quartz seems to support the thesis that the reaction is mostly quartz dependent - no new bands were visible in the IR spectrum (Online Resource 1, S.M. 5). Despite being the simplest proteinogenic amino acid, glycine is probably one of the most problematic to examine, due to the co-existence of three different polymorphs (Chernobai et al. 2007; Ferrari et al. 2003). The spectra of glycine-before and after the reaction are represented in Fig. 3, with arrows indicating new visible bands. The full spectrum is presented in Online Resource 1, S.M. 6. However, as the distinction between polymorphic transitions and structural alteration, caused by electric discharge, appears to be unachievable at the current stage of experiment, no further examination of data was attempted.

For these reasons, the experiment was performed with alanine, as it has only one polymorphic structure. The comparison between spectra before and after the reaction is shown in Fig. 4again the biggest changes are indicated by arrows and full spectra are presented in Online Resource 1, S.M. 7. It seems that only small amount of alanine underwent the reaction, as the obtained spectrum is largely the spectrum of the substrate. Apart from the appearance of new bands, the relative intensities of the original bands also do change.

Therefore, in order to evaluate the changes in intensity, integration of all of the bands (data not shown) and normalization to two bands $\left(650\right.$ and $\left.2,986 \mathrm{~cm}^{-1}\right)$ was performed. The bands, that the spectra were normalized to, seemed to be invariable to the reaction, with respect to band position and shape. Only changes greater than $10 \%$ of the starting intensity were taken into account and analysed (Online Resource 1, S.M. 8).

After the reaction, 10 new bands at approx. 1330, 1038, 931, 897, 798, 694, 682, 589, 537 and $506 \mathrm{~cm}^{-1}$, appeared, showing the creation of new reaction products of alanine. It was assumed that the reaction proceeded with the occurrence of oxygen radicals, since they are very probable to be created in a water solution.

According to Johnson et al. (1989), reaction of amino acids with water-based free radicals, results in formation of aldehydes and keto acids. Therefore, mainly pyruvic acid and acetaldehyde should be formed from alanine. This is supported by the appearance of new bands at 506, 589, 681, 798 and 1,330 $\mathrm{cm}^{-1}$ (Kleiner et al. 2008; Reva et al. 2001; Spectroscopy online, cited $1128,2012)$. This would also provide an explanation for some of the increased intensities. For more detailed data and list of references, refer to Online Resource 1, S.M. 8. 
Fig. 3 FTIR-ATR spectra of the glycine-before (red) and after the reaction (blue), in different spectral ranges: a $3,300-1,900 \mathrm{~cm}^{-1}$ and $\mathbf{b}$ $1,700-400 \mathrm{~cm}^{-1}$. Spectra were offset for clarity
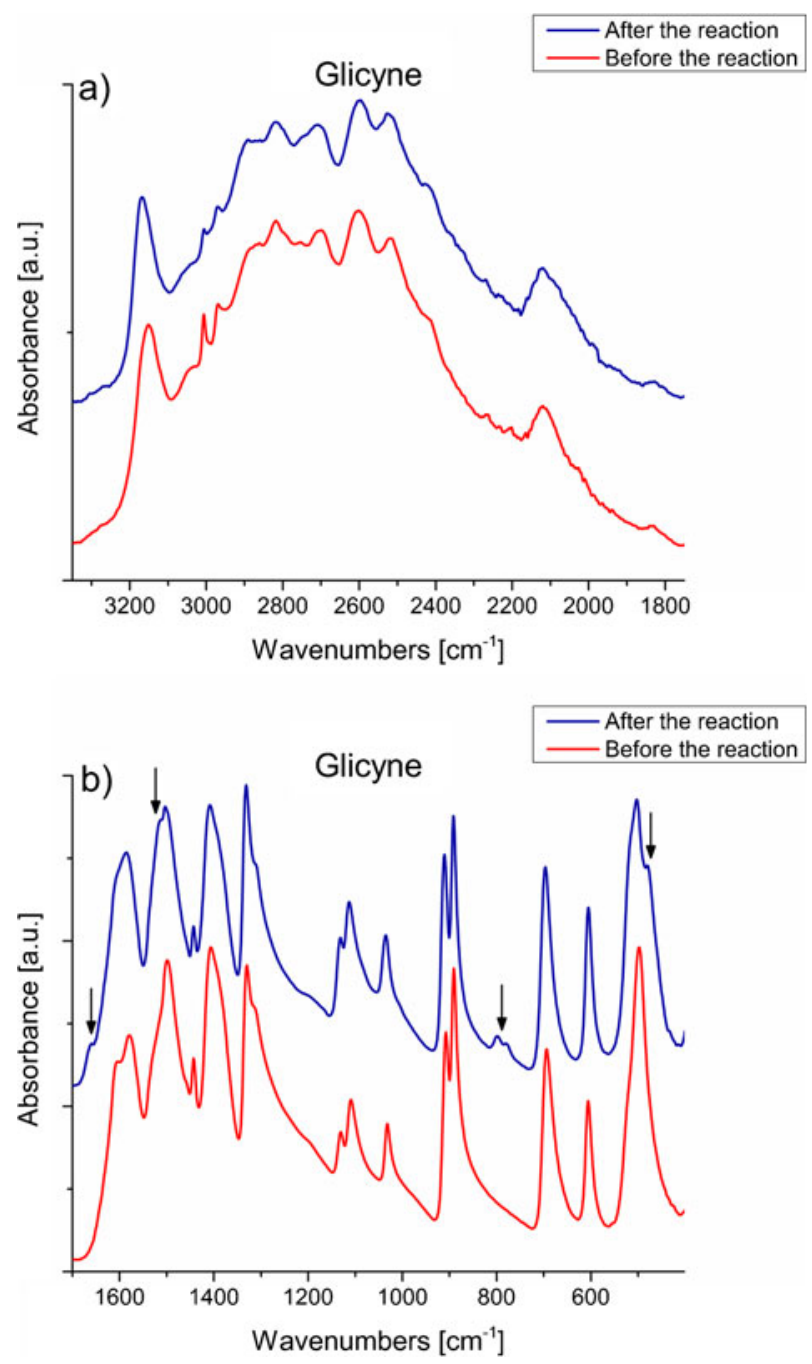

Since the $\mathrm{NH}_{2}$ group of amino acids should also be easily and readily oxidized to NO or $\mathrm{NO}_{2}$, formation of nitro- based species cannot be excluded. This would be supported by new bands at 694, 897 and $1,039 \mathrm{~cm}^{-1}$ (Spectroscopy online, cited 1128,2012 ) and some of the changing intensities (Barthes et al. 2002; Gerakines et al. 2012; Minkov et al. 2010; Rozenberg et al. 2003; Wang et al. 1971) (Online Resource 1, S.M. 8).

Further and indisputable explanation of an ongoing reaction and identification of its products would require performing more specific analyses, namely mass spectroscopy or chromatography. However, from this very preliminary experiment it can be concluded that formation of dipeptides or any polypeptides is highly unlikely in the studied environment. Treatment of quartz with an electric discharge creates a radical rich, mostly oxidizing environment. The main compounds identified are products of degradation of alanine and if any peptide synthesis occurred, the products would be destroyed in a similar fashion. Therefore, close proximity of quartz along with electric discharge does not create a suitable platform for creation of proteins. 
Fig. 4 FTIR-ATR spectra of the alanine-before (red) and after the reaction (blue), in different spectral ranges: a $3,300-2,000 \mathrm{~cm}^{-1}$ and $\mathbf{b}$ $1,700-300 \mathrm{~cm}^{-1}$. Spectra were offset for clarity
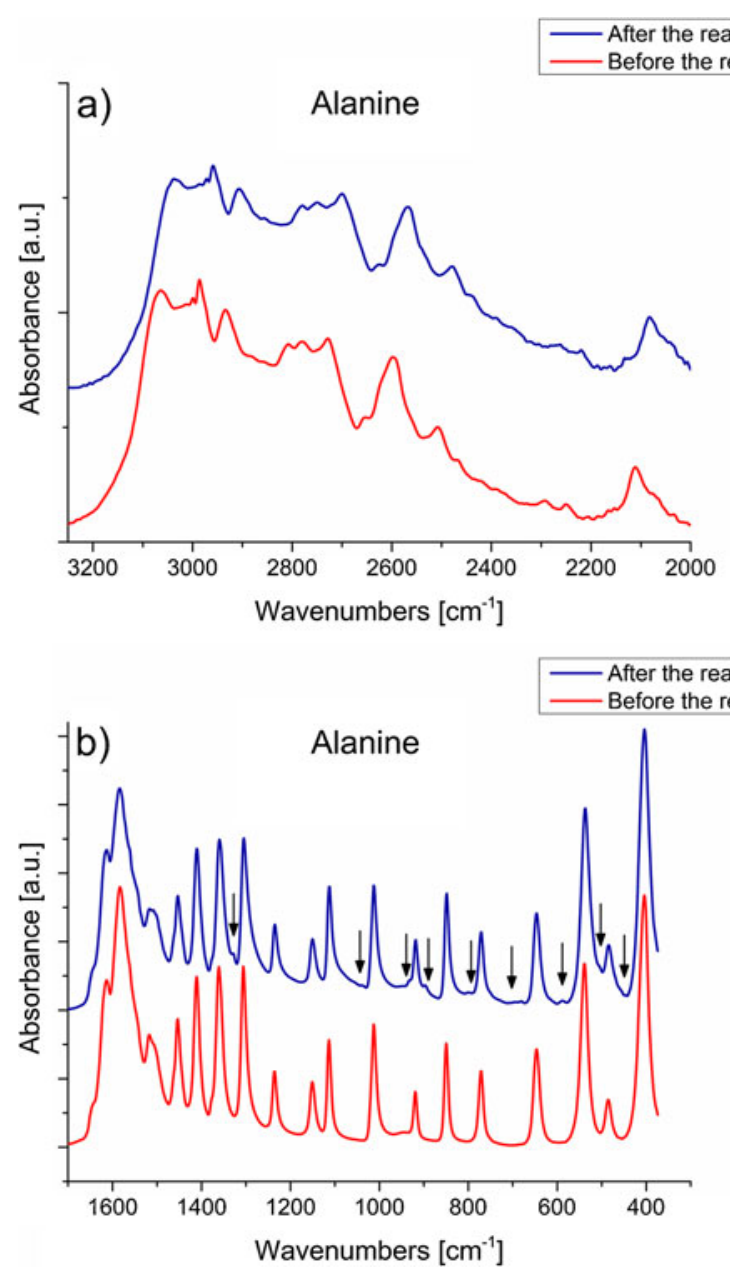

\section{Conclusion}

The performed experiments and presented results have proven that quartz, under the influence of electric discharge, has the potential to stimulate chemical transitions and reactions of amino acids, namely glycine and alanine. The main catalysing factor of such reaction seems to be free radicals - their generation had been proven by DPPH-based UVVIS examination. Although the results are preliminary, along with the literature (Johnson, et al. 1989), they suggest transition of amino acids into aldehydes and keto acids, which bands were found in the spectra of the products. However, further confirmation by performing additional tests is required in order to specifically define the products. Therefore, it is concluded that quartz, along with electric discharge does not create a suitable environment for creation of peptides.

Acknowledgments The authors would like to thank Prof. Malgorzata Baranska (Faculty of Chemistry, Jagiellonian University) for making it possible to perform all the experiments and their results presented here. 
Open Access This article is distributed under the terms of the Creative Commons Attribution License which permits any use, distribution, and reproduction in any medium, provided the original author(s) and the source are credited.

\section{References}

Apopei AI, Buzgar N, Buzatu A (2011) Raman and infrared spectroscopy of kaersutite and certain common amphiboles. Analele Stiintifice ale Universitatii “Al I Cuza” din Iasi Seria Geologie 57(2):35-58

Barthes M et al (2002) Breathers or structural instability in solid L-alanine: a new IR and inelastic neutron scattering vibrational spectroscopic study. J Phys Chem A 106:5230-5241

Bobrowski A, Holtzer M (2010) Oznaczanie zawartości SiO2 w piasku chromitowym metodą spektroskopii w podczerwien. Arch Foundry Eng 10(2):19-22

Chernobai GB et al (2007) Temperature effects on the IR spectra of crystalliine amino acids, dipeptides, and polyamino acids. I. Glycine. J Struct Chem 48(2):332-339

Damm C, Peukert W (2009) Kinetics of radical formation during the mechanical activation of quartz. Langmuir 25:2264-2270

Ferrari ES et al (2003) Crystallization in polymorphic systems: the solution-mediated transformation of $b$ to a glycine. Cryst Growth Des 3(1):53-60

Gerakines PA et al (2012) In situ measurements of the radiation stability of amino acids at $15-140 \mathrm{~K}$. Icarus 220:647-659

Hazen RM (2006) Mineral surfaces and the prebiotic selection and organization of biomolecules. Am Mineral 91:1715-1729

Hlavay J et al (1978) Characterization of the particle size and the crystallinity of certain minerals by ir spectrophotometry and other instrumental methods - II. Investigations on quartz and feldspar. Clays Clay Miner 26(2):139-143

Johnson GRA, Nazhat NB, Saadalla-Nazhat RA (1989) Reactions of the hydroxyl free radical with copper(II)amino-acid complexes in aqueous solution. J Chem Soc Faraday Trans I 85(3):677-689

Kazarian SG, Chan KLA (2006) Applications of ATR-FTIR spectroscopic imaging to biomedical samples. Biochim Biophys Acta 1758:858-867

Kleiner I et al (2008) Assignment, fit, and theoretical discussion of the 10 band of acetaldehyde near $509 \mathrm{~cm}$. J Mol Spectrosc 225:214-229

Lambert JF (2008) Adsorption and polymerization of amino acidson mineral surfaces: a review. Orig Life Evol Biosph 38:211-242

Minkov VS, Chesalov, Yu A, Boldyreva EV (2010) A study of the temperature effect on the IR spectra of crystalline amino acids, dipeptids, and polyamino acids. VI. L-alanine and dl-alanine. J Struct Chem 51(6):1052-1063

Pawlikowski M (2012) Atomic structural templates of the earliest life on earth: vibration and lightning experiments with quartz and amino acids. [book auth.]. In: Joseph S (ed) Genesis - in the beginning. precursors of life, chemical models and early biological evolution. s.1, vol 22. Springer, Netherlands, pp 171-177

Pross A (2004) Causation and the origin of life. Orig Life Evol Biosph 34:307-321

Reva ID et al (2001) Combined FTIR matrix isolation and ab initio studies of pyruvic acid: proof for existence of the second conformer. J Phys Chem A 105(19)

Rozenberg M et al (2003) Low-temperature Fourier transform infrared spectra and hydrogen bonding in polycrystallineL-alanine. Spectrochimica Acta A 59:3253-3266

Sahni M, Locke BR (2006) Quantification of hydroxyl radicals produced in aqueous phase pulsed electrical discharge reactors. Ind Eng Chem Res 45(17)

Saikian BJ, Parthasarathy G, Sarma NC (2008) Fourier transform infrared spectroscopic estimation of crystallinity in $\mathrm{SiO} 2$ based rocks. Bull Mater Sci 31(5):775-779

Shneider H (1978) Infrared spectroscopic studies of experimentally shock-loaded quartz. Meteoritics 13(2)

Shoval S (1991) A new method for measuring the crystallinity index of quartz by infrared spectroscopy. Mineral Mag 55:579-582

Spectroscopy online. [Online] [Cited: 11 28, 2012.] http://www.spec-online.de/

Ueda S et al. (2009) Development of compact ozonizer using wire to plane electrodes. Washington DC. 17th IEEE International Pulsed Power Conference, Vol 5386175. pp 994-998

Wang CH, Storms RD (1971) Temperature dependent raman study and molecular motion in L-alanine single crystal. J Chem Phys 55(7)

Wróbel TP et al (2011) Imaging of lipids in atherosclerotic lesion in aorta from ApoE/LDLR mice by FT-IR spectroscopy and Hierarchical Cluster Analysis†. Analyst 136(5247)

Wróbel TP et al (2012) Attenuated total reflection Fourier transform infrared (ATR-FTIR) spectroscopy of a single endothelial cell. Analyst 137(4135) 